\title{
COM \\ Free Bright Conversations - meaningful participatory activities for the communication of the Sustainable Development Goals (SDGs)
}

\section{Manuel Ballatore and Marie Bartsch}

\begin{abstract}
Political, economic and social actors have begun to implement the 17 SDGs (UN 2030 Agenda) to build a desirable future for everyone. To reach this goal, a mix of systemic alteration and individual change is needed. "Free Bright Conversations" is a dialogue-based science communication event developed at MUSE-Science Museum in Trento that focuses on people's engagement with sustainable development. The paper describes the format and provides an evaluation based on preliminary data collected on two occasions. The authors conclude that participatory science communication furthers involvement with our common, sustainable future.
\end{abstract}

Keywords

DOI

Community action; Environmental communication; Science centres and museums

https://doi.org/10.22323/2.19040801

Submitted: 28th October 2019

Accepted: 22nd July 2020

Published: 3rd August 2020

Introduction

In September 2015 the UN General Assembly launched the 2030 Agenda for sustainable development [A/RES/70/1], a programmatic document for wide-ranging transformation. The Agenda contains 17 Sustainable Development Goals (SDGs) that deal with the world's critical, interrelated issues: poverty, hunger, health, education, gender equality, water and sanitation, energy, decent work, industry and innovation, inequalities, cities and communities, consumption and production, climate, biodiversity and ecosystems; besides peace, justice and partnership.

The awareness for environmental deterioration and the corresponding social consequences have recently increased, as initiatives like the Fridays for Future movement show. However, society still exhibits inertness. While concrete systemic change is without a doubt necessary, we have chosen to focus on the individual due to interesting dynamics that have been observed, among others, by Sheila Jasanoff. In her paper "A new climate for society" [2010] she has explored the gap between experts' warnings and society's response. Jasanoff has reported 
dissonances in the dimensions of time, space, community, and polity. Due to common "goals and determinants" [Yohe et al., 2007], we are able to apply her theoretical insights to all areas of sustainable development.

According to Jasanoff [2010], an explanation for expert-society gaps in the dimension of time can be found in the temporal distance between the life of the individual and experts' climate scenarios. For the sake of persuasion, these are often set in the far future, in which negative consequences of the unsustainable lifestyle are visible in their extreme form. However, a future laying considerably beyond the individual's lifespan becomes unrelatable. Similar mechanisms apply to the dimension of space. Here, the engagement of society with sustainability is complicated through the missing ability to "sense" climate change or structural inequalities in a concrete spot [Jasanoff, 2010]. However, global and complex threats can gain meaning by being translated into situated routines and stories, relating systemic processes to specific change [Ungar, 2000]. A viable way to tickle these two problem dimensions is to invite people's active participation in imagining and negotiating a desirable world with others. Thereby, a shared vision may be constructed that can be acted upon [Anderson, 2010; Yusoff and Gabrys, 2011].

In the community dimension, Jasanoff [2010] criticizes the use of "survival language" in the communication of sustainability. Feelings of hopelessness will only lead to disengagement in the long run. Instead, sharing practical knowledge and innovative ideas for change might turn action-taking into a hopeful common endeavour. Linking the dimension of polity and knowledge, the scholar also argues that concepts of science, technology, and nature may be discussed based on multiple, culturally embedded ways of knowing [Jasanoff, 2005]. Science communication that provides various publics with the platform to meet, construct meaning and articulate concepts, can contribute proactively to a sustainable future. Environmentally and socially sustainable communities will be advanced through common practices and shared responsibilities [Davies, 2016].

Communication for sustainable development and the Sustainable Development Goals (SDGs) is a hot topic on multiple levels, from policy to academia [Mulholland, 2019]. Science Museums are significant conversation leaders for their vocation as places for informal learning, and their affinity with environmental issues, science and technology, public awareness and future-related scenarios. Worldwide, museums' efforts concerning the UN 2030 Agenda have been formally stated [Science Centre World Summit, 2017]. The MUSE-Science Museum has declared its investment in this area since 2015 [Bernardi et al., 2015; Tombolato and Ballatore, 2017; Ballatore and Rusci, 2018; Ballatore and Eriksson, 2018; Tombolato, 2018]. Among the activities of the SDGs communication and education program, Free Bright Conversations (FBCs) have been developed as a master thesis project in partnership with the University of Trento and Maastricht University.

In this paper, we aim to investigate the potential of dialogic science communication to diffuse knowledge about the SDGs and motivate action-taking. We have designed the format FBCs for public space, to engage people with the UN 2030 Agenda through dialogues, in which educative and 'cogenerative' approach are matched, responsibility and self-efficacy are fostered. First, the format design will be introduced with its main values, materials, and method. FBCs are relational 
experiences that allow people with diverging concepts on sustainability to share and discuss their knowledge in a personal manner. Second, the data of two occasions where FBCs have been run are reported. The analysis is organized around the following themes: "Sustainable lifestyle and Familiarity with SDGs", "Perception of the Conversation" and "Engagement and Diffusion". Following, we discuss the results according to the theoretical framework provided by Jasanoff [2010]. We conclude that participatory interventions can indeed challenge the gap between the expert and the individual in the time/space and the community/polity dimension. Finally, we provide an outlook for further research.

Free Bright Conversations: format design
The format offers an opportunity to articulate knowledge and ideas about a sustainable future, in the framework of the SDGs. FBCs function as dialogic events in the tradition of participatory science communication. Therefore, among the 3 strategies of engagement, a conversation with the facilitator represents the core activity. By holding up a speech bubble sign, the facilitator invites a conversation about sustainable development, lifestyle, habits, believes and hopes about the future. FBCs are devised to be social experiences that connect people with different concepts and values with each other. The activity moves the debate on sustainability away from the digital into real-life time and space. Dialogues take place in public locations and can occur with individual persons or in groups. The participants depend on the chosen location, of which market squares, science festivals, university lobbies or metro stations are examples.

The facilitators' role in the conversation is crucial. By keeping the tone personal and emotional and by using common language, they produce a friendly atmosphere. This, in turn, may help the participants to gain confidence. Like that, the participant can become an active contributor during the FBCs and knowledge is produced in a joint effort. All collocutors are equals and learn from each other while sharing their ideas, tips, vulnerabilities, and doubts. While it is not the aim of FBCs to arrive at shared conclusions, being forced to deal with conflicting concepts and values can contribute to the complexity of conversations. The audience-centred and embedded approach considers ideas, experiences, and routines of the public, while the 17 SDGs function as the framework to structure the talks and to give a sense of global identity and relevance. Sharing knowledge and thoughts in a hopeful way creates a different frame for debates on sustainability and is valuable in two ways. First, it encourages action-taking. Secondly, it makes more probable that participants will engage with the discourse on own initiative, in their private circle of friends and family.

FBC centre on an imaginative exercise that relates global challenges to specific individual events and possible actions. Further, the concepts of society, science, technology, and nature are openly negotiated as well as awareness built for the sustainability concept as depicted in the 17 SDGs. As mentioned above, the format contains 3 forms of engagement of which talking with the facilitator is one. As an alternative or in combination with the conversation, a live poll triggers people's involvement with the format. Finally, the format includes also a quick survey to be completed afterwards. Other than as evaluation for us, the survey also functions as a moment of reflection for the participants. 


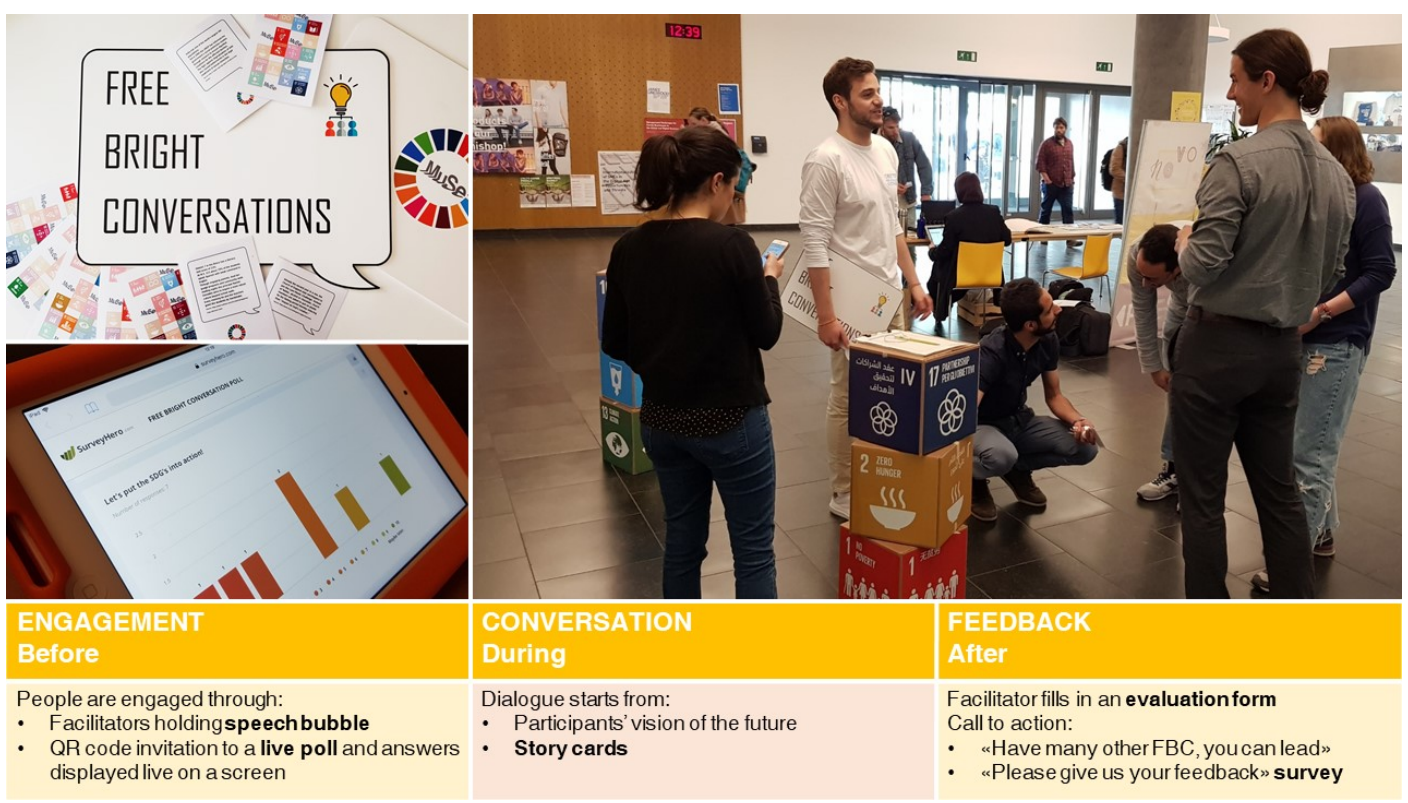

Figure 1. Free Bright Conversations schematic overview.

FBCs format contains (Figure 1):

1. Live poll - Few questions to catch the curious who scan the QR code.

2. Conversation with a facilitator - Speech bubble shows availability for conversation.

3. Survey - 3-minute survey with 17 questions.

1. Live poll. During the event, participants have the opportunity to join an on-site poll by scanning a QR code with their smartphones. Posters with the code are attached to the wall or handed out by the facilitators. Conversation participants and passers-by alike may join the poll. The results are displayed live on a tablet or other screen and are also accessible via an e-mail link.

The goal is to attract the participants' attention and give them the chance to voice their opinion directly through five quick answers to the questions described in Figure 2. At the same time, the responses provide data about their understanding of the concept of sustainability detached from the conversations. With the poll, we have aimed for casual answers, which would be less subject to self-control. The questions have been formulated in a playful tone but with a serious twist to invite the participants to critically reflect on own sustainable behaviour.

2. Conversation. Most of the times facilitators will choose passers-by as conversation partners at random. Other times, they might be approached by curious participants themselves. The facilitators should be easy to speak to, friendly and curious. Although they are scientifically briefed and equipped with data, the task is rather to encourage the public to share their interests, beliefs, and knowledge about sustainability. Depending on the participants' contributions and attitude, conversations do not need to touch upon scientific facts at all but might 
deal exclusively with tangible consequences of sustainability in daily life. FBCs create a space free of judgment, striving to provoke curiosity and create awareness. There is no time limit to the discussions as long as they stay focussed and appreciated. While a certain assertiveness might be necessary, participants and their schedule should be respected.

There are two ways to build a conversation, and combinations of both approaches are possible. In both versions, the facilitator guides the participants and uses the SDGs whenever helpful to bridge subjects or clarify relations. The first is a talk which develops around the invitation to the participants to imagine the future and articulate their vision. Following, the vision is discussed in detail and ideas revised on how to contribute to the future the participant would desire. In the second version, conversations start picking a story card. Here, participants are asked to translate a brief story about the SDGs into their geographical area and personal practices. By connecting global goals to personal thoughts and routines in the here and now, the format evokes emotional engagement. The cards are designed to bridge potential emotional and cognitive gaps and create a common ground on which participants can build fruitful conversations. The stories on future tech, schooling the community, changing actors in the sustainability debate, urban ecology and social inclusion, can be interpreted from multiple angles (Annex 1 in the supplementary material).

After each conversation, facilitators fill in a paper evaluation form (Figure 2) and note relevant aspects of the conducted talk. For the sake of the smallest disruption during the intervention, the questionnaire has been designed to be informative but short, taking less than a minute to complete. These forms document the event from the facilitators' perspective, which is compared to observations and participants' feedback (Annex 2 in the supplementary material).

3. Survey. The participants are given the chance to voice their opinion on the FBCs and the time to continue their reflection on the SDGs. At the end of each conversation, participants are invited to leave their e-mail address on a list so they may be contacted afterwards. In the mail, they find the request to answer a survey of 17 questions by following an embedded link. Each of the participants receives a personalized link bound to one device only. Next to general information about age, occupation, and nationality, the questions (Figure 2) are formulated to capture the participants' assessment of FBCs. Specifically, it is measured how successful the format is in creating an atmosphere which is personal and comfortable, revealing the relevance of SDGs to the participants' life and strengthening the feeling of agency in sustainable development.

Data collection and results
After designing the activity following the theoretical framework, we have scheduled the format to assess its performance and receive feedback. The evaluation explores if the format fulfils the requirements for a valuable personal experience and science communication action: we examine how successful the event works in spreading knowledge about the SDGs and, since FBCs aim to encourage people to act, we present an analysis of how likely it is that participants recognize their agency. The collected sets of data provide insights into the future that the participants have imagined, their information on sustainable behaviour and possibly feelings of efficacy. 


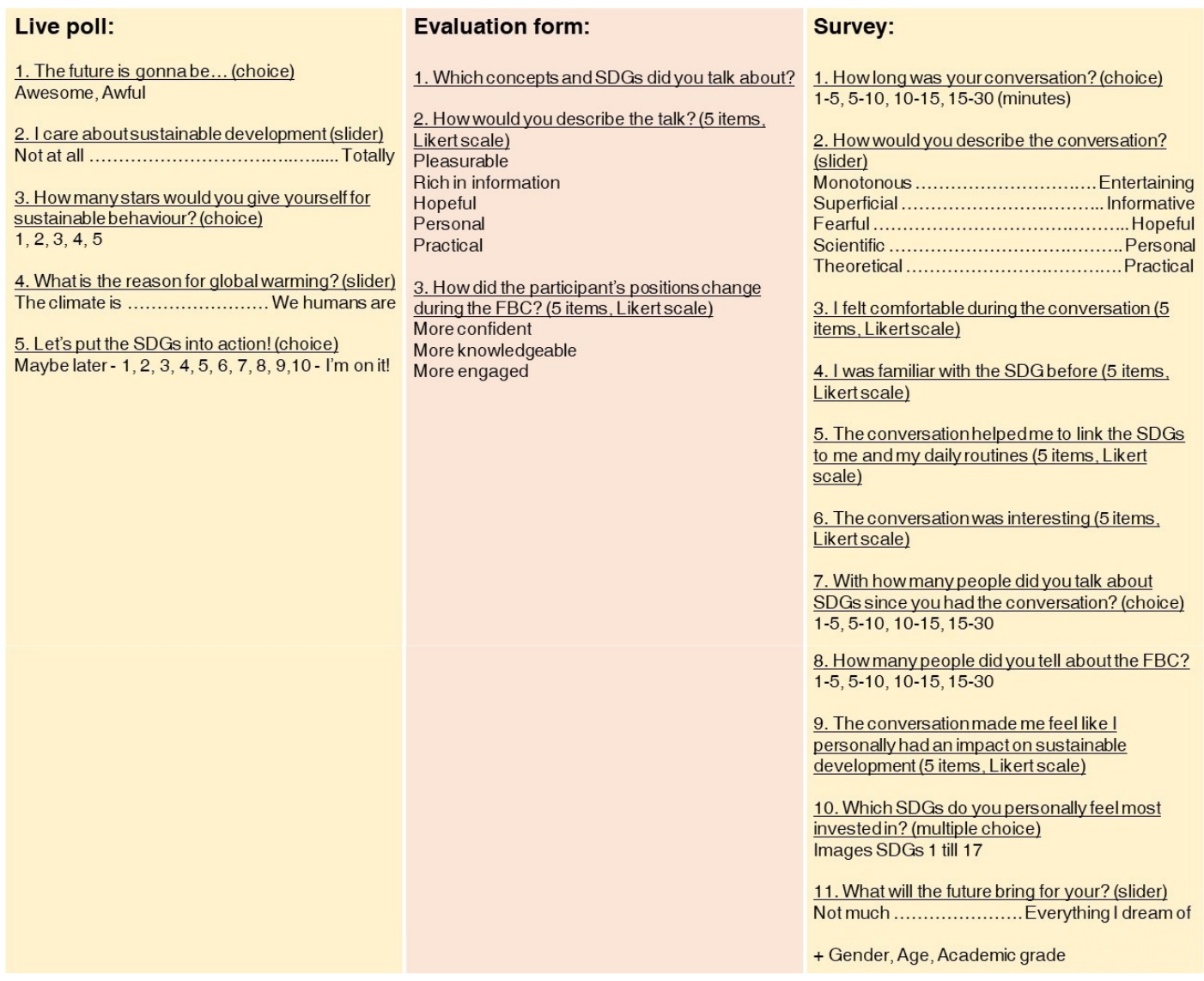

Figure 2. Specific content of the live poll, facilitators' evaluation form and participants' survey.

Data has been collected at the Sustainability Festival at the Free University of Bozen-Bolzano (April 2019) and in the urban park, Le Albere, in the city of Trento (July 2019). In the first case, FBCs were part of the festival program and took place during the coffee-breaks, thus representing an application in a well-defined context (specific public). In the second case, the activity has been run as street action outside any organized event (park visitors as the general public). Three science communicators were present on-site, the authors of this article in the role of observer and facilitator, plus another facilitator.

In Bozen-Bolzano, 22 passers-by have filled in the poll and the results have been displayed on a tablet, freely accessible during the event. Answers have been registered anonymously to allow also for controversial statements. There have been participants who could not access the poll as they could not operate the QR scanning option on their mobile phones. In Trento, where a total of 8 people has joined the poll, the outside location made it impossible to display the results live. However, participants received a link to them. To map and compare frameworks between the participants and the science mediators, also the latter ones have completed the poll questions via a separate link. The results of the participant poll can be found in Annex 2 in the supplementary material, together with the poll results of the facilitators.

As concerning the conversations, Facilitators engaged in 15 talks in Bozen-Bolzano and 8 in Trento. All the participants received the invitation for the survey, but only 
12 from Bozen-Bolzano and 5 from Trento have completed the survey about their experience at a later point. A reminder to complete the questionnaire has been sent two days before the survey has been closed for respondents. The raw results can be found in Annex 3 in the supplementary material.

The main results are shown regarding three thematic areas.

1. Sustainable lifestyle and familiarity with SDGs. Both in Bozen-Bolzano and Trento, participant survey results have shown that a high percentage of the sample size has consisted of students from Italian universities. Being part of a normed educational system, it can be assumed that the majority of our collocutors have come in contact with current paradigms on sustainability, climate science and the Anthropocene. For example, a great majority of participants have voiced the opinion that humans are responsible for global warming. Survey results from Bozen-Bolzano have shown that participants have previously known of the SDGs. Participants in Trento have felt undecided about their familiarity with the SDGs before joining the FBCs. The poll answers from both events have confirmed the participants' awareness of sustainability and their willingness for critical self-reflection. All collocutors have expressed interest in sustainability but have rated own sustainable behaviour rather modestly. Participants in Trento have shown to be tendentially negative about the level of sustainability they live by. According to our results, participants have felt most invested in SDG 12 (Responsible Consumption and Production) and SDG 13 (Climate Action). This engagement with environmental topics has also been mirrored in the actual conversations. The talk evaluations forms have shown for both Bozen-Bolzano and Trento that SDG 4 (Quality Education), SDG 11 (Sustainable Cities and Communities) and SDG 12 are of greatest interest to the sample size.

2. Perception of the conversations. Participants' and facilitators' perception of the FBCs has differed in nuances in both editions. On average, all collocutors in Bozen-Bolzano have agreed that the talks have been entertaining, rich in information and hopeful. Taking the objectives of the format into consideration, participants have rated the conversation slightly better than the facilitators in these regards. In Trento, participants have even strongly agreed that the talks have been entertaining and rich in information, while facilitators have rated them average entertaining and informative. All parties have found the FBCs to provide a hopeful atmosphere and have been undecided about the direct, practical value of the conversation. However undecided, the participants have rated the practical value still slightly higher than the facilitators. While all parties have agreed that the FBCs have been personal, the participants in Trento have been slightly less convinced than the facilitators. This hesitation has also been expressed by the participants in Bozen-Bolzano, who have displayed uncertainty about the personal depth of their FBCs. Although group conversations have initially been perceived as less personal, they have shown greater progress in awareness of sustainable development and engagement, according to the facilitators. Meanwhile, the facilitators have found the conversations of Bozen-Bolzano to be personal and practical. According to the survey data, they have felt very comfortable and very interested in the Bozen-Bolzano edition, respectively very comfortable and interested during the second edition in Trento. 
3. Engagement and diffusion. In the live polls, most participants have indicated that they care about sustainable development and have thereby implied, at least cognitive, engagement with the topic. The majority of all parties have also stated that they were occupying themselves with putting the SDGs into action. Herein participants in Trento have appeared to be more motivated than the ones in Bozen-Bolzano. More than half of participants in Bozen-Bolzano and Trento have expressed positive expectations of the future, and so have all facilitators. The latter ones have voiced the opinion that, in Bozen-Bolzano as well as in Trento, participants have gained knowledge about the SDGs, more confidence in expressing their opinion and even left the FBCs more engaged with the sustainability debate. This has appeared in the FBCs evaluation forms. In fact, participants whose confidence had needed most encouragement, have eventually cogenerated the most personal meaning on the SDGs, according to the talk evaluation forms. Additionally, participants have agreed in both editions that the format has helped them to link the SDGs to their everyday routines and has shown them they personally have an impact on sustainable futures. Participants in the Trento edition expressed themselves on an average more positive than the ones in Bozen-Bolzano. In the participant feedback surveys, it has been indicated by both editions' participants that SDGs and FBCs have been mentioned to a similar number of people in the period between the event and the completion of the survey.

FBCs make a valuable contribution to the debate on science communication and engagement with the SDGs. The results, interpreted in the light of the theoretical framework, support that participatory interventions can challenge the science-society gap in the time and space, and the community and polity dimension, as we show below.

Time and space. Imagining and articulating their future had been intended to support participants' engagement with sustainable development. Concerning this aim, facilitators have indeed reported that the participants have been more motivated at the end of the FBCs. This might be interpreted as proof that highlighting the possibility for multiple futures and choices [Yusoff and Gabrys, 2011] can aid feelings of efficacy. The future gains meaning and options through imaginative engagement in participatory interventions. According to Anderson [2010], imaginaries guide material actions through expectations. Participants have agreed that FBCs had helped them to relate their actions to the sustainable development goals and had made them feel like they personally had an impact. This can be interpreted as that the future imaginary represents a successful tool in participatory interactions to exemplify that the individual needs to act upon the future. Facilitators have directed the participants from the articulation of a wish or fear of the future to the formulation of a plan of action on an individual or collective level. Like this, the imaginary future may gain details and emotions.

On the other hand, participants have been investing cognitive and emotional energy into correlating the global and the local with the help of the story cards. Jasanoff [2010] has mentioned how environmental activism has historically originated from the desire to protect one's home and local nature. Blatt [2013] affirmingly refers to the three-step model of environmental identity development, in which encounters with local nature are often leading to awareness equalling the 
first level. According to the results of our research, all our participants have already passed this step in their development. Thus, during FBCs, the facilitators have proceeded immediately to the second phase which is connected to the feeling of empowerment. To tackle the SDGs on a personally relevant level, we have talked about everyday routines to make the SDGs actionable. In keeping with "Think globally, act locally", the metaphor of the home as the smallest unit for change [Yusoff and Gabrys, 2011] has been used to exemplify SDGs 11-12, which are of special interest to participants. FBCs have dealt with tips and difficulties in sustainable consumption and regional food production alternatives, also specifically in the context of the shared household.

Special attention has been paid to carving out in which areas the participants had already started to act responsibly against their environment. Following Blatt [2013], a positive appraisal of sustainable behaviour reinforces environmental identity. Additionally, it is a strategy to overcome potential frictions in other social spheres whose discouragement weakens sustainable self-conception. Rating their sustainable behaviour in the poll has invited critical self-reflection of the participants. Blatt [2013] has pointed out that some harbour greater ability for critical reflection than others when their environmental identity is put to a test. The face-to-face conversation allows the facilitator to change their approach according to the participant's needs and interests. FBCs have been positively evaluated by participants on stressing personal impact and linking SDGs to individual routines. That implies that through the possibility to respond to the individual collocutor, FCB strengthens feelings of empowerment necessary for environmental identity development.

Community and polity. The "fight for survival" language leads to resignation instead of a sense of kinship and responsibility [Jasanoff, 2010]. According to O'Neill and Nicholson-Cole [2009], negative emotions, like fear or shock, lose effect quickly. To encourage collective action, science communication can provide meeting places to share positive, practical, and empowering messages and build new communities. The participants overall have had positive memories of the event and had felt comfortable and interested. They have perceived the conversation as hopeful, which clearly shows that participatory interventions are a suitable medium to create positive frames for sustainability. The excess work, which is required to explain oneself, convince others and negotiate concepts, will result in a better understanding of the topics discussed [Colucci-Gray et al., 2013].

The tension between the national politics of knowledge-making and the need for global policymaking has been increasing [Jasanoff, 2004]. Global threats require political structures inclusive to citizens and their culturally diverse practices of knowing, according to Jasanoff [2004]. As educational institutions, science centres and museums contribute to the political education of society. That is why they should not hesitate to provide places for dynamic interaction and deliberation, without the constraint for consensus. According to Davies, McCallie et al. [2009], in deliberative science communication activities, all parties can test ideas and respond to others without feeling the need to see them as political enemies.

Towards the end, facilitators have asked participants to initiate FBCs themselves. Our results show a quantitative correlation between the conversations that participants have been having about the FBCs and the SDGs. This means that 
participants who have shared knowledge about the SDGs would have mentioned also the FBCs. There are two options. Either the FBCs have functioned as the frame to discuss the SDGs in which case this has happened in an encouraging and hopeful way, as we have discussed before. Or the FBCs have been mentioned as an experience to support an overarching argumentation, adding to the knowledge on the SDGs. The results imply that participatory interventions are effective in sustaining talk about sustainable development in a pleasurable way, in the private sphere.

Conclusion

Science communication helps citizens to "construct identities, solidify social relations, shore up organizational cultures [and] engage in political debate" [Davies and Horst, 2016].

With science at their core, science centres and museums have taken up the duty to inform society about the 17 sustainable development goals [Science Centre World Summit, 2017]. Based on our research, we have concluded that cultural instruments can contribute to sustainable futures by furthering society's engagement with sustainable development and the SDGs. Participatory activities support individual and collective meaning-making of scientific facts by embedding them in thick experiences. This has been shown on the case of the specific dialogic event Free Bright Conversations (FBCs). We have researched how participatory interventions in science communication strengthen engagement in sustainable feeling and thinking by challenging the problems of time, community, polity and space.

In the dimension of time, we conclude that the future gains meaning and options through imaginative exercises during the participatory intervention. Participants have agreed that FBCs have helped them to relate own actions to sustainable development and have made them feel like they personally have an impact. Creating imaginaries with participants is effective in triggering awareness for a desirable future, which needs to be actively shaped. In turn, this has reintroduced feelings of agency and empowerment, which is needed to motivate action-taking. In the realm of space, participatory interventions resolve problems of scale and personal engagement with global challenges by translating the systemic to the local and specific. Participants have been investing cognitive and emotional energy into translating global into authentic, meaningful stories, which has made the geographically distant palpable. This has opened up opportunities to reassess environmental identities and the personal space for improvement within an intimate, trustful conversation with responsive mediators.

Participatory interventions are an excellent opportunity for sharing experiences and own practices, and for collective sense-making. During FBCs, especially the group conversations have engaged participants in lively discussions on sustainability. The mediators have succeeded in framing sustainable development conversations as hopeful and encouraging. As a pleasurable experience, FBCs have shown to be effective in sustaining talk about the SDGs in other social spheres or even framing them. This way relevance for the topic is created beyond educational and scientific entities, which is an important prerequisite for strengthening the sustainable community. 
Concerning polity, we find that participatory interventions ideally function as a platform for opinion building on controversial topics and articulation of own ideas. The power over the structure and timing of the activity and the knowledge produced is shared between participants and facilitators. In fact, we noticed how FBCs have given participants the space to muster the courage to express their thoughts. In conclusion, dialogic events provide alternative places for deliberation and the development of democratic capacities.

Secondary research, based on bigger sample size, would corroborate the significance of our findings by confirming the tendencies described. Additionally, the effects of FBCs on participants could be more explicitly related to the format, if questionnaires would be filled out before and after the talk. Moreover, it needs to be investigated if participatory activities like FBCs can influence the construction of shared concepts and common values in society and how this process can be best guided. Especially interesting are the power dynamics between facilitators and participants and the nature of knowledge that is generated in open formats like FBCs. We suggest that further studies also examine how formats like the FBCs relate to parts of the SDGs that do not centre on climate change. In the surge of protests for Black Lives Matter - how can this mix of personal narratives and scientific communication on the SDGs help to encounter the complexity of systemic inequality?

Acknowledgments Free Bright Conversations is part of the Project TASK-Toward A Sustainable Know-how, founded by the Autonomous Province of Trento through the grant "I Comunicatori STAR della Scienza".

The authors thank the Kikero Unibz- Cultural Student Association Free University of Bozen-Bolzano, and Viktor Vasilev and Giorgio Palpacelli for their contribution as facilitators.

Additionally, we would like to express gratitude to Prof Emanuela Bozzini (University of Trento, Department of Sociology and Social Research) and Dr Lorenzo Beltrame for the thesis supervision. Thanks to Marjolein Asselt (University Maastricht) for her helpful comments on the thesis.

\section{References}

Anderson, B. (2010). ‘Preemption, precaution, preparedness: anticipatory action and future geographies'. Progress in Human Geography 34 (6), pp. 777-798. https://doi.org/10.1177/0309132510362600.

Ballatore, M. and Eriksson, A. E. (2018). 'La notte più colorata di sempre. L'edizione 2018 della notte dei ricercatori'. Natura Alpina 69, pp. 319-320.

Ballatore, M. and Rusci, E. (2018). 'L'umanità oltre il limite. Misurare il nostro impatto sul pianeta'. Natura Alpina 69, pp. 311-314.

Bernardi, M., Menegon, M., Negra, O. and Pallaveri, A. (2015). 'Estinzioni. Storie di catastrofi ed altre opportunità'. Natura Alpina 66, pp. 1-208.

Blatt, E. N. (2013). 'Exploring environmental identity and behavioral change in an Environmental Science course'. Cultural Studies of Science Education 8 (2), pp. 467-488. https://doi .org/10.1007/s11422-012-9459-2. 
Colucci-Gray, L., Perazzone, A., Dodman, M. and Camino, E. (2013). 'Science education for sustainability, epistemological reflections and educational practices: from natural sciences to trans-disciplinarity'. Cultural Studies of Science Education 8 (1), pp. 127-183. https://doi.org/10.1007/s11422-012-9405-3.

Davies, S. (2016). 'Participation as pleasure: citizenship and science communication'. In: Remaking participation: science, environment and emergent publics. Ed. by J. Chilvers and M. Kearnes. Abingdon, U.K.: Routledge, pp. 162-177. https://doi.org/10.4324/9780203797693.

Davies, S., McCallie, E., Simonsson, E., Lehr, J. L. and Duensing, S. (2009). 'Discussing dialogue: perspectives on the value of science dialogue events that do not inform policy'. Public Understanding of Science 18 (3), pp. 338-353. https://doi.org/10.1177/0963662507079760.

Davies, S. R. and Horst, M. (2016). Science communication: culture, identity and citizenship. London, U.K.: Palgrave Macmillan. https://doi.org/10.1057/978-1-137-50366-4.

Jasanoff, S. (2004). ‘Ordering knowledge, ordering society'. In: States of knowledge: the co-production of science and social order. Ed. by S. Jasanoff. London, U.K.: Routledge, pp. 13-45. https://doi.org/10.4324/9780203413845.

- (2005). 'Civic epistemology'. In: Designs on nature: science and democracy in Europe and the United States. Princeton, NJ, U.S.A.: Princeton University Press, pp. 247-271. https://doi .org/10.1515/9781400837311-014.

- (2010). 'A new climate for society'. Theory, Culture \& Society 27 (2-3), pp. 233-253. https://doi .org/10.1177/0263276409361497.

Mulholland, E. (2019). 'Communicating sustainable development and the SDGs in Europe: good practice examples from policy, academia, NGOs, and media'. In: ESDN quarterly report 51. Vienna, Austria, pp. 3-21.

O'Neill, S. and Nicholson-Cole, S. (2009). " Fear Won't Do It". Promoting positive engagement with climate change through visual and iconic representations'. Science Communication 30 (3), pp. 355-379. https://doi.org/10.1177/1075547008329201.

Science Centre World Summit (2017). On the role of Science Centre and Science Museum worldwide in support of the United Nations Sustainable Development Goals. URL: https://scws2017.org/tokyo_protocol/.

Tombolato, D. (2018). 'A caccia di strategie (sostenibili). L'Italia, l'agenda 2030 e il MUSE'. Natura Alpina 69, pp. 309-310.

Tombolato, D. and Ballatore, M. (2017). '2030. Obiettivi di sviluppo sostenibile e progetto TASK al MUSE'. Natura Alpina 68, pp. 197-198.

Ungar, S. (2000). 'Knowledge, ignorance and the popular culture: climate change versus the ozone hole'. Public Understanding of Science 9 (3), pp. 297-312. https://doi.org/10.1088/0963-6625/9/3/306.

Yohe, G. W., Lasco, R. D., Ahmad, Q. K., Arnell, N. W., Cohen, S. J., Hope, C., Janetos, A. C. and Perez, R. T. (2007). 'Perspectives on climate change and sustainability'. In: Climate change 2007: impacts, adaptation and vulnerability. Contribution of working group II to the fourth assessment report of the intergovernmental panel on climate change. Ed. by M. L. Parry, O. F. Canziani, J. P. Palutikof, P. J. van der Linden and C. E. Hanson. Cambridge, U.K.: Cambridge University Press, pp. 811-841. URL: https://www.ipcc.ch/site/as sets/uploads/2018/02/ar4-wg2-chapter20-1.pdf.

Yusoff, K. and Gabrys, J. (2011). 'Climate change and the imagination'. Wiley Interdisciplinary Reviews: Climate Change 2 (4), pp. 516-534.

https://doi.org/10.1002/wcc.117. 
Manuel Ballatore (IT) is a science communicator at MUSE-Science Museum in Trento (IT) leading a post-doc project on the UN 2030 Agenda. Previously Earth Science reasearcher and science teacher, he is interested in formal and informal science education, sustainability, complex systems, audience development, and art \& science communication. E-mail: manuel.ballatore@libero.it.

Marie Bartsch (DE) recently obtained a master's degree in European Studies of Society, Science and Technology from the University of Maastricht (NL) after taking her specialisation in Science Communication at the University of Trento (IT). Her research interest are society, art \& communication, science \& technology, and museum studies. E-mail: marie.bar7sch@gmail.com.

How to cite

\section{Supplementary material}

Ballatore, M. and Bartsch, M. (2020). 'Free Bright Conversations - meaningful participatory activities for the communication of the Sustainable Development Goals (SDGs)'. JCOM 19 (04), N01. https:/ / doi.org/10.22323/2.19040801.

Available at https://doi.org/10.22323/2.19040801.

Annex 1: Story cards.

Annex 2: Results of the live poll, collected from 22 participants in Bozen-Bolzano and 8 in Trento; results from the facilitators are also reported.

Annex 3: Results of the survey, collected from 12 participants in Bozen-Bolzano and 5 in Trento. 